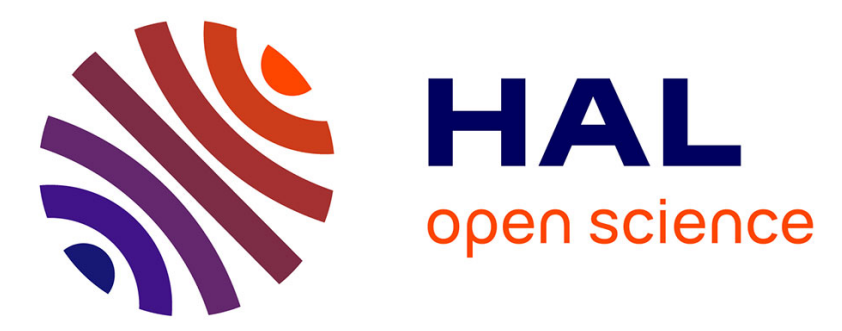

\title{
Polarization-Time Coded OFDM for PDL Mitigation in Long-Haul Optical Transmission Systems
}

Elie Awwad, Yves Jaouën, Ghaya Rekaya-Ben Othman, Erwan Pincemin

\section{To cite this version:}

Elie Awwad, Yves Jaouën, Ghaya Rekaya-Ben Othman, Erwan Pincemin. Polarization-Time Coded OFDM for PDL Mitigation in Long-Haul Optical Transmission Systems. European Conference on Optical Communications, Sep 2013, London, United Kingdom. pp.P3.4. hal-00991715

\section{HAL Id: hal-00991715 https://hal.science/hal-00991715}

Submitted on 15 May 2014

HAL is a multi-disciplinary open access archive for the deposit and dissemination of scientific research documents, whether they are published or not. The documents may come from teaching and research institutions in France or abroad, or from public or private research centers.
L'archive ouverte pluridisciplinaire HAL, est destinée au dépôt et à la diffusion de documents scientifiques de niveau recherche, publiés ou non, émanant des établissements d'enseignement et de recherche français ou étrangers, des laboratoires publics ou privés. 


\title{
Polarization-Time Coded OFDM for PDL Mitigation in Long-Haul Optical Transmission Systems
}

\author{
Elie Awwad ${ }^{(1)}$, Yves Jaouën ${ }^{(1)}$, Ghaya Rekaya-Ben Othman ${ }^{(1)}$, Erwan Pincemin ${ }^{(2)}$ \\ (1) Institut Telecom / Télécom ParisTech, CNRS UMR 5141, 75634 Paris, France, \\ elie.awwad@telecom-paristech.fr \\ (2) France Telecom, Orange Labs, 22307 Lannion Cedex, France
}

\begin{abstract}
We experimentally demonstrate the potential of Polarization-Time codes in mitigating PDL in long-haul transmissions. The Silver code exhibits the best performance (2dB Q-penalty reduction at a PDL of $6 d B$ ). Moreover, for inline PDL, it improves the mean Q-factor by $0.6 d B$ while significantly narrowing the variance of the corresponding distribution.
\end{abstract}

\section{Introduction}

Polarization-multiplexed (PolMux) coherent optical systems combined with DSP techniques has established itself as a key solution for current and future ultra-high bit rate optical transmissions ${ }^{1}$. Among the linear impairments affecting these systems, Polarization Dependent Loss (PDL) was the remaining unsolved performance-degrading effect until very recently, when advanced coding techniques have been suggested $^{2}$. Space-Time (ST) codes have been designed for wireless MIMO communications in order to exploit all the degrees of freedom of these systems. The Golden and Silver codes are the best performing codes designed for MIMO systems with 2 emitting and 2 receiving antennas in a random fading environment. They have been adapted to the optical PolMux channel and are referred to as Polarization-Time (PT) codes. PT codes have proven to be efficient in mitigating $\mathrm{PDL}^{2,3}$.

PT coding consists in sending different linear combinations of symbols $S_{1}, S_{2}, S_{3}, S_{4}$ (i.e., QPSK symbols) on 2 polarizations $\left(\mathrm{pol}_{1}, \mathrm{pol}_{2}\right)$ during 2 symbol times $\left(T_{1}, T_{2}\right)$. The corresponding transmitted codeword matrix $X$ is given $b^{2}$ :

$$
\begin{aligned}
& \left.X=\left[\begin{array}{ll}
f_{1}\left(S_{1}, S_{2}, S_{3}, S_{4}\right) & f_{3}\left(S_{1}, S_{2}, S_{3}, S_{4}\right) \\
f_{2}\left(S_{1}, S_{2}, S_{3}, S_{4}\right) & f_{4}\left(S_{1}, S_{2}, S_{3}, S_{4}\right)
\end{array}\right]\right\} \begin{array}{ll}
p_{1} \\
\text { pol }_{2}
\end{array} \\
& T_{1} \underbrace{}_{T_{2}}
\end{aligned}
$$

The Golden and Silver codes are full-rate, redundancy-free codes, i.e. the transmitted bit rate is the same as for a PolMux system with independent QPSK symbols sent on each polarization, whereas the Alamouti code is a half-rate code. Their coding gains are acquired when Maximum-Likelihood (ML) decoding is performed at the receiver side. Besides, the use of OFDM insures a single-tap frequency-domain channel equalization of reasonable complexity.

Preliminary investigations of PDL mitigation using PT codes were limited to a linear channel with lumped PDL. In this paper, we examine the ability of PT codes to mitigate PDL in a $1000 \mathrm{~km}$ -



Fig. 1: Experimental setup. (ECL: External Cavity Laser, AWG: Arbitrary Waveform Generator, MUX: Multiplexer, AO: AcoustoOptical Modulator, PS: Polarization Scrambler, OBPF: Optical Band-Pass Filter, ASE: Accumulated Spontaneous Emission source, LO: Local Oscillator, OSA: Optical Spectrum Analyzer, OSC: Tektronix 50GS/s Oscilloscope)

optical link with distributed PDL taking into account the interactions of PDL with distributed ASE noise ${ }^{4}$ and non-linear effects ${ }^{5}$.

\section{Experimental setup}

The transmission scheme, shown in Fig. 1, is composed of a PolMux OFDM transmitter, a recirculating loop and a dual-polarization coherent receiver. A total effective bit rate of $25 \mathrm{Gbps}$ is achieved, leading to $100 \mathrm{Gbps}$ if a multi-band OFDM approach ${ }^{6}$ is considered. The OFDM signals transmitted on two orthogonal polarization states consist in 256 subcarriers including 194 data subcarriers and 10 pilot subcarriers for common phase error estimation. In order to compare the performance of the PT codes, we alternatively modulate the data subcarriers with QPSK (No-coding), Silver-, Golden- and Alamouti- coded symbols. To insure the same spectral efficiency for all 
codes, Alamouti-coded subcarriers use 16-QAM symbols whereas the Golden and Silver codewords are built using QPSK symbols. An 18-sample cyclic prefix is appended to each OFDM symbol to absorb inter-symbolinterference. A 22-symbols training sequence is inserted each 100 OFDM symbols for time synchronization, frequency offset compensation and channel estimation. PT coding correlates the polarization-multiplexed symbols. Hence, four DACs are necessary to generate two complex baseband OFDM signals. Having a unique AWG (Tektronix 7122B) with two outputs, we will consider OFDM symbols satisfying the Hermitian symmetry property. This constraint does not affect our analysis knowing that the complex conjugate of the subcarriers will be treated independently of the original subcarriers at the receiver side. The signals have a $\sim 9 \mathrm{GHz}$ bandwidth and the raw bit rate is $36 \mathrm{Gbps}$ taking into account the different transmission overheads. Up-conversion to the optical domain is done by two single-drive Mach-Zehnder modulators (MZM). Then the two optical signals are combined by a Polarization Beam Combiner and multiplexed among seven $50 \mathrm{GHz}$-spaced wavelengths. The path difference between the two tributaries of the PolMux OFDM signal is accurately compensated.

The transmission line consists in a recirculating loop which contains 2 spans of $100 \mathrm{~km}$ of SMF, a PDL element of $2 \mathrm{~dB}$, a polarization scrambler and an optical band-pass filter. After additional ASE noise loading at the receiver, the desired wavelength $(\lambda=1542.9 \mathrm{~nm})$ is filtered and the signal is detected with a dual-polarization coherent receiver. An External cavity laser ECL $(\Delta v=100 \mathrm{kHz})$ is used as an optical carrier at the transmitter and as a local oscillator at the receiver. A real-time Tektronix oscilloscope samples the received signal at $50 \mathrm{GSa} / \mathrm{s} .2 \cdot 10^{6}$ samples $\left(\sim 10^{5}\right.$ bits/code) are recorded for each channel realization and offline-processing ${ }^{1,7}$

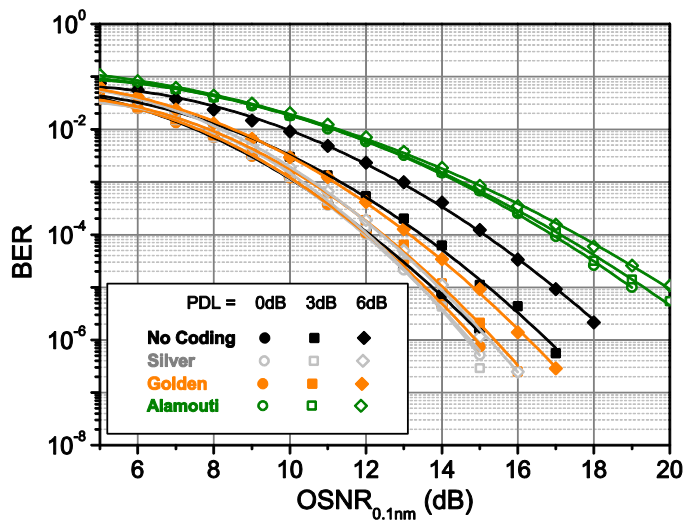

Fig. 2: BER curves versus OSNR $\mathrm{O}_{0.1 \mathrm{~nm}}$ with $\mathrm{PDL}=0,3$, $6 \mathrm{~dB}$ for the different schemes: no coding, Silver, Golden and Alamouti codes. ending with $M L$ decoding is carried to measure the BER and the corresponding Q-factor in $\mathrm{dB}$ $\left(B E R=0.5 \operatorname{erfc}(Q / \sqrt{2})\right.$ and $\left.Q_{d B}=20 \log _{10}(Q)\right)$.

\section{Comparison of OSNR degradation with PDL}

Before transmitting the PT-coded OFDM signals in the recirculating loop, we conduct preliminary measurements of the BER evolution as a function of the OSNR for 3 different PDL values at the transmitter: 0,3 and $6 \mathrm{~dB}$. An attenuator is inserted in one branch of the PolMux transmitter to emulate the worst OSNR degradation of one polarization tributary ${ }^{5}$. The loop is replaced by a polarization scrambler and the signal is then combined with an ASE noise source. The measured BER curves are plotted in Fig. 2. We note firstly that the Silver code performs better than the Golden code and secondly that the performance of the Alamouti code is independent of the PDL value. The observed coding gains have been theoretically explained in ${ }^{8}$. In Fig. 3, for a PDL ranging from 0 to $6 \mathrm{~dB}$, we show the $\mathrm{Q}$-factor penalties obtained with QPSK, Silver and Golden codes with regard to a PDL-free case at an OSNR of $13 \mathrm{~dB}$. We see that the Silver code performs the best reducing the Q-penalty by $2 \mathrm{~dB}$ for a $\mathrm{PDL}=6 \mathrm{~dB}$.

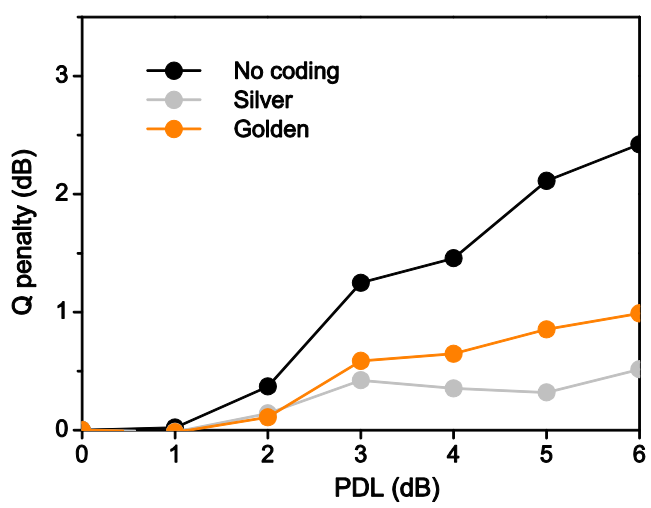

Fig. 3: Q-penalty versus PDL at $\mathrm{OSNR}_{0.1 \mathrm{~nm}}=13 \mathrm{~dB}$ with regard to a PDL-free scenario.

\section{Lumped PDL}

After these preliminary tests, we will transmit the PT-coded OFDM in the recirculating loop of Fig. 1 and replace the in-line PDL element by an attenuator having the same insertion loss. PDL is emulated at the transmitter side as in the previous section. BER measurements are carried out after a $1000 \mathrm{~km}$-transmission for a span input power of the modulated channel ranging from -15 to $5 \mathrm{dBm}$. For each input power, up to 50 realizations are considered depending on the target BER. The obtained bellshaped curves are plotted in Fig. 4 for the QPSK and Silver-coded schemes at a PDL of 0,3 and $6 \mathrm{~dB}$. We firstly note that all curves have their optimum operating point at $-3 \mathrm{dBm}$. However in presence of PDL, the uncoded scheme severely suffers $(Q=10.5 \mathrm{~dB}$ at $-3 \mathrm{dBm}$ for $P D L=6 \mathrm{~dB})$ 
while the Silver code completely compensates the penalty at $\mathrm{PDL}=3 \mathrm{~dB}$ and nearly all penalty at $P D L=6 \mathrm{~dB}(\mathrm{Q}=12.3 \mathrm{~dB}$ at $-3 \mathrm{dBm})$. Moreover, an important observation is that the $\mathrm{PT}$-coded OFDM does not induce any extra penalties in the non-linear propagation regime.

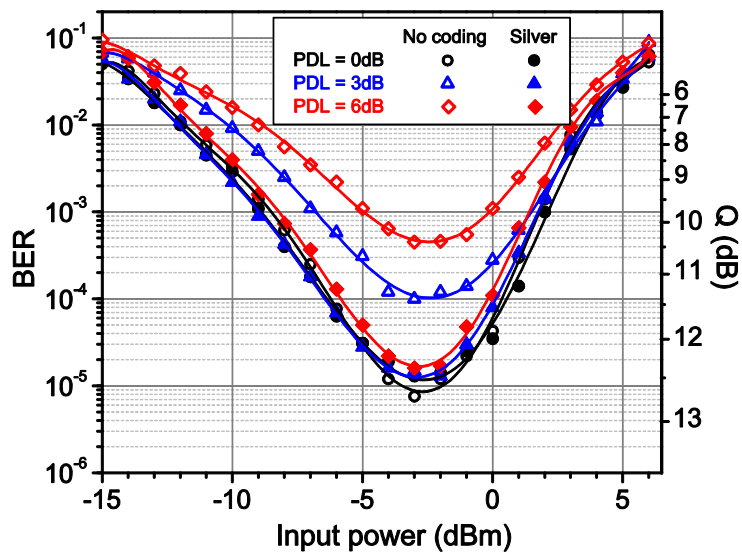

Fig. 4: Comparison of BER evolution versus launched input power after $1000 \mathrm{~km}$ for the Silver-coded and QPSK schemes (PDL at the transmitter $=0,3,6 \mathrm{~dB}$ ).

\section{Distributed in-line PDL}

PDL being a distributed effect induced by in-line components along the optical transmission link, we are interested in testing the capability of codes in mitigating in-line PDL. Therefore, we remove the emulated $\mathrm{PDL}$ at the transmitter and insert a PDL element of $2 \mathrm{~dB}$ into the loop. Then, we extract the Q-factor distribution for the different coding schemes by measuring 2000 Q-factor values at the optimum operating point corresponding to an input power of $-3 \mathrm{dBm}$. The measured OSNR at the receiver is $12 \mathrm{~dB}$ after additional ASE noise loading in order to evaluate the complete Q-factor distribution.

The obtained Q-factor distributions are shown in Fig. 5. After 5 loops, we measure PDL using the estimated $2 \times 2$ channel matrices and obtain a Maxwellian probability distribution function of PDL with a 4.2dB mean (the expected theoretical mean being $4 \mathrm{~dB}^{9}$ ). The inset in Fig. 5 shows the measured probability distribution and the fitted Maxwellian distribution.

We notice that the $\mathrm{Q}$-factor distribution for the QPSK-coded subcarriers has a mean of $11.2 \mathrm{~dB}$ and a standard deviation (std) of $0.55 \mathrm{~dB}$ whereas the mean Q-factor of the Silver- and Golden- coded subcarriers is $11.8 \mathrm{~dB}$ (the same observed value for the PDL-free case when OSNR $=12 \mathrm{~dB}$ ) and the $\mathrm{Q}$-factor distributions are narrower. Note, as well, that the Silver code gives a distribution slightly narrower than the one obtained with the Golden code: std of $0.35 \mathrm{~dB}$ and $0.37 \mathrm{~dB}$ respectively. Regarding the Alamouti code, we observe a mean $Q$-factor of $8.3 \mathrm{~dB}$ due to the use of 16-QAM symbols
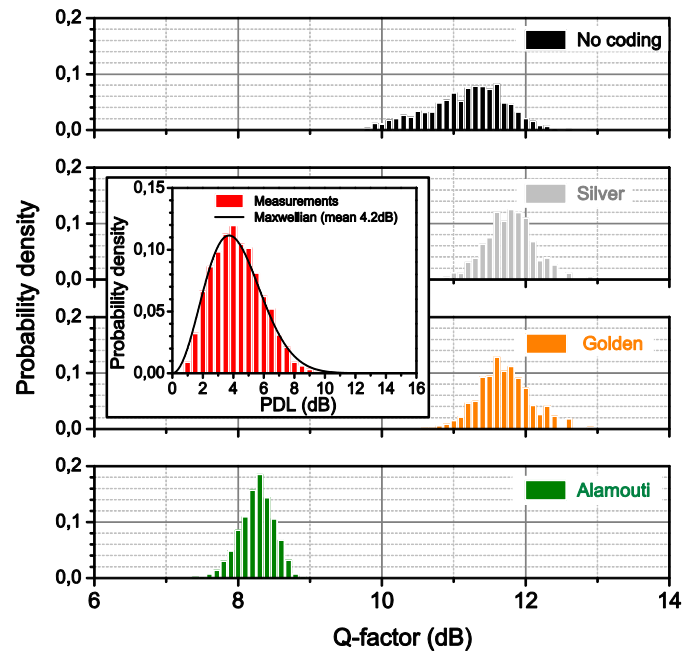

Fig 5: Q-factor probability distributions after $1000 \mathrm{~km}$ for $P_{\text {in }}=-3 \mathrm{dBm}\left(O S N R_{0.1 \mathrm{~nm}}=12 \mathrm{~dB}\right)$. Inset: probability distribution of PDL measured with 2000 realizations.

instead of QPSK to guarantee the same spectral efficiency. However, its Q-factor distribution is the narrowest of all with a std of $0.24 \mathrm{~dB}$. This observation can be explained by the powerful orthogonal structure of the Alamouti codeword matrix that makes its performance independent of the amount of accumulated PDL in the link ${ }^{8}$. The observed small variance of its $Q$-factor distribution can be explained by the increased sensitivity of 16-QAM modulation to phase noise and non-linear effects.

\section{Conclusions}

We have experimentally demonstrated, for the first time, the potential of PT codes to mitigate PDL in long-haul optical links, as well as their resistance to non-linear effects. An enhanced performance evaluated through Q-factor measurements was observed when PT codes are used: a narrower Q-factor distribution is obtained and the mean Q-factor is improved.

\section{Acknowledgements}

This work has been partially supported by the Celtic-Plus SASER-SIEGFRIED project.

\section{References}

[1] W. Shieh et al., Opt. Express 15, pp. 99369947 (2007).

[2] S. Mumtaz et al., Proc. ICC'10, pp.1-5(2010).

[3] S. Mumtaz et al., Proc. SPPcom'11, paper SPWB6 (2011).

[4] C. Xie, Proc. OFC'10, paper OWe6 (2010).

[5] O. Vassilieva et al., Opt. Express 19, pp. B357-B362 (2011).

[6] J. Karaki et al., Proc. ECOC'12, paper P4.17 (2012).

[7] S. Jansen et al., JLT vol. 26, pp. 6-14 (2008).

[8] E. Awwad et al., ICC'13, accepted (2013).

[9] A. Mecozzi et al., PTL vol.14, pp. 313-316 (2002). 